\title{
Are we going to end up with many distinct genomic syndromes in psychiatry?
}

\author{
Dan Rujescu
}

Published online: 15 March 2011

(C) Springer-Verlag 2011

The knowledge resulting from the availability of the complete sequence of the human genome, the systematic identification of genetic variants throughout the genome and the development of parallel genotyping technology established the conditions that brought about the current revolution in our ability to probe the genome for identifying disease genes. Genomic studies have opened a window into the biology of common complex diseases and have yielded several genes showing strong association with complex diseases or traits including Crohn's disease, diabetes and many others. These studies revealed genes involved in pathogenesis and identified entirely unexpected disease pathways. This is of utmost importance given that this knowledge can eventually translate into the development of better treatment or even cure.

A major challenge in medicine is to understand genetic, molecular and cellular mechanisms underlying mental disorders including schizophrenia, which involves complex genetic and environmental determinants. The last few years brought up a series of studies in schizophrenia which substantially advanced the knowledge on the genetic causes and had a major impact on the field. Especially, genome-wide studies on copy number variants (CNVs) raised highest interests. A CNV is a segment of DNA in which copy number differences have been found. Humans usually have two copies of each autosomal region, one per chromosome. This may vary for particular genetic regions due to deletions or duplications. Spontaneous (or inherited) CNVs can therefore be identified in a hypothesis-free genome-wide approach. These techniques have shown that

D. Rujescu $(\bowtie)$

Department of Psychiatry, Ludwig-Maximilians-University,

Nußbaumstr. 7, 80336 München, Germany

e-mail: Dan.Rujescu@med.uni-muenchen.de a much higher number of CNVs exists in humans than previously recognised. In a genome-wide search for CNVs associating with schizophrenia performed by the SGENE consortium, a population-based sample was used to identify de novo CNVs by analysing 9,878 transmissions from parents to offspring. Three deletions at $1 \mathrm{q} 21.1$ (OR $=$ 14.8), $15 \mathrm{q} 11.2(\mathrm{OR}=2.7)$ and $15 \mathrm{q} 13.3 \quad(\mathrm{OR}=11.5)$ showed association with schizophrenia [1] and could be replicated several times $[2,3]$.

Interestingly, some of the discovered deletions in schizophrenia have been shown to associate with a number of unexpected phenotypes, including minor dysmorphic features, abnormal EEG, significant expressive language deficits and a spectrum of neuropsychiatric impairments that include epilepsy, ADHD, autism spectrum disorder and cognitive impairment varying from moderate to mild learning disability. Thus, CNV carriers need to be carefully characterised regarding clinical signs and symptoms, as well as cognitive and behavioural features across diagnostic boundaries (for review see [4]).

The discovery of CNVs associated with neurodevelopmental disorders has raised a number of fascinating new clinical and scientific questions, especially concerning the phenotypic boundaries between major neurodevelopmental disorders as they are currently classified, their modes of inheritance, the implications of this new information for diagnostics and genetic counselling, the genetic and environmental factors that determine the penetrance and expressivity of CNV-associated neurodevelopmental phenotypes, and finally the relationship between human behaviour itself and human genomic evolution.

It is plausible that a fraction of individuals that carry such CNVs will be defined as distinct "genomic disorders" in the coming years. This gives hope for the broad spectrum of neurodevelopmental disorders that appear to be 
partially made up from multiple rare disorders. In particular, knowledge on these CNVs could provide opportunities for genetic counselling in families in which CNVs segregate, improve the diagnostic assessment, classification and prognostic estimates for a group of rare syndromic CNS diseases, which would not be possible by any other means and be useful in the development of disease specific therapies, as has been the case for Fragile X syndrome, a rare $\mathrm{X}$-linked disorder in which model mice have led to the development of glutamate antagonists as novel therapies.

It will be necessary to go all the way from the CNV to its biochemical cellular systems and behavioural impact in the carriers. Furthermore, the development of in vitro (cell culture) and transgenic mouse models, in which synthetic deletions or duplications, ranging from multiple genes to single exons, will be crucial. These mice and cells must be characterised in detail for pathophysiological features of the disease to generate models that can be used to pilot pharmacological interventions based on the nature of the primary lesion. These findings may only be the beginning and genetic approaches may have a major impact on new classifications of psychiatric syndromes. In this line a "decade for psychiatric diseases" has been proclaimed in the fist Nature issue of this decade given that research in schizophrenia has already shown fascinating discoveries making them worth to be mentioned on the front page of another 2010 Nature issue.

New hope rises with ongoing whole-genome sequencing efforts that are under way. A recent study based on wholegenome DNA sequencing data from 185 human genomes mapped over 22,000 deletions and 6,000 additional CNVs. Over half of them could be mapped to nucleotide resolution, which facilitates analysing their origin and functional impact. This study shows that CNVs are abundant in humans, differing from other forms of variation in extent, origin and functional impact [5]. This data and further data to be generated shortly may serve as a resource for future $\mathrm{CNV}$ association studies. Ultimately, whole-genome sequencing of a large number of patients and controls will be performed in the near future and will hopefully add to the understanding of neurodevelopmental disorders including schizophrenia.

\section{References}

1. Stefansson H, Rujescu D, Cichon S, Pietiläinen OP, Ingason A, Steinberg S, Fossdal R, Sigurdsson E, Sigmundsson T, BuizerVoskamp JE, Hansen T, Jakobsen KD, Muglia P, Francks C, Matthews PM, Gylfason A, Halldorsson BV, Gudbjartsson D, Thorgeirsson TE, Sigurdsson A, Jonasdottir A, Jonasdottir A, Bjornsson A, Mattiasdottir S, Blondal T, Haraldsson M, Magnusdottir BB, Giegling I, Möller HJ, Hartmann A, Shianna KV, Ge D, Need AC, Crombie C, Fraser G, Walker N, Lonnqvist J, Suvisaari J, Tuulio-Henriksson A, Paunio T, Toulopoulou T, Bramon E, Di Forti M, Murray R, Ruggeri M, Vassos E, Tosato S, Walshe M, Li T, Vasilescu C, Mühleisen TW, Wang AG, Ullum H, Djurovic S, Melle I, Olesen J, Kiemeney LA, Franke B, Kahn RS, Linszen DH, van Os J, Wiersma D, Bruggeman R, Cahn W, de Haan L, Krabbendam L, Myin-Germeys I, Sabatti C, Freimer NB, Gulcher JR, Thorsteinsdottir U, Kong A, Andreassen OA, Ophoff RA, Georgi A, Rietschel M, Werge T, Petursson H, Goldstein DB, Nöthen MM, Peltonen L, Collier DA, St Clair D, Stefansson K (2008) Large recurrent microdeletions associated with schizophrenia. Nature 455(7210):232-236

2. ISC (2008) Rare chromosomal deletions and duplications increase risk of schizophrenia. Nature 455:237-241

3. Kirov G, Grozeva D, Norton N, Ivanov D, Mantripragada KK, Holmans P, International Schizophrenia Consortium, Wellcome Trust Case Control Consortium, Craddock N, Owen MJ, O'Donovan MC (2009) Support for the involvement of large copy number variants in the pathogenesis of schizophrenia. Hum Mol Genet 18(8): 1497-1503

4. Mitchell KJ (2010) The genetics of neurodevelopmental disease. Curr Opin Neurobiol 21(1):197-203

5. Mills RE, Walter K, Stewart C, Handsaker RE, Chen K, Alkan C, Abyzov A, Yoon SC, Ye K, Cheetham RK, Chinwalla A, Conrad DF, Fu Y, Grubert F, Hajirasouliha I, Hormozdiari F, Iakoucheva LM, Iqbal Z, Kang S, Kidd JM, Konkel MK, Korn J, Khurana E, Kural D, Lam HY, Leng J, Li R, Li Y, Lin CY, Luo R, Mu XJ, Nemesh J, Peckham HE, Rausch T, Scally A, Shi X, Stromberg MP, Stütz AM, Urban AE, Walker JA, Wu J, Zhang Y, Zhang ZD, Batzer MA, Ding L, Marth GT, McVean G, Sebat J, Snyder M, Wang J, Ye K, Eichler EE, Gerstein MB, Hurles ME, Lee C, McCarroll SA, Korbel JO, 1000 Genomes Project (2011) Mapping copy number variation by population-scale genome sequencing. Nature 470(7332):59-65 